\title{
Study on Testing Technology of Creepage Distances and Clearances in Safety Standards for Electric Toys
}

\author{
Yan-Yan SUO ${ }^{1, a}$, Li-Juan YIN ${ }^{1}$, Cheng-Bo LIANG ${ }^{1}$ and Bei-Bei XU1, 2 \\ ${ }^{1}$ Shenzhen Entry- Exit Inspection and Quarantine Bureau, 518045, Shenzhen, Guangdong, China \\ 2Shenzhen Academy of Inspection and Quarantine, 518010, Shenzhen, Guangdong, China \\ tjusuo@126.com
}

Keywords: Creepage distance, Clearance, Insulation, Pollution degree, Electric toy.

\begin{abstract}
In electric toy tests, the creepage distance and clearance tests are related to techniques of various fields and there are no specific measurement paths and rules in relative standards, which results in the big difference in test results. In this paper, the technical requirements of the creepage distance and clearance are compared and analysed according to IEC62115 and GB19865 respectively and the effects of the pollution degree and material insulation on the measurement are studied and then the paths and rules of measurement are suggested to make sure the test results are more accurate.
\end{abstract}

\section{Introduction}

Lots of test data show that there are various safety dangers or more than $20 \%$ electric toys, including the shortage of creepage distance and clearance result from poor design or incorrect assembling which may cause high surface temperature to burn children or even cause electric shock or short circuit to bring about fire to make hazards to the users' health and properties. There are many return and recall cases of the exported toys for the safety problems.

Clearance is the shortest distance in air between two conductive parts or between a conductive part and the accessible surface, which will avoid transient overvoltage and repetitive peak voltage

Creepage distance is the shortest distance along the surface of insulation between two conductive parts or between a conductive part and the accessible surface, which will test the resistance of the insulation under a given operation voltage and pollution grade.

There are safety requirements for the clearance and the creepage distance in electric toys in many toy standards such as IEC62115, EN62115, GB19865 and so on, but there are no specific test methods. These two tests cover much technical knowledge and many technological difficulties, which usually results in very different results on the same sample to make wrong assessment.

In order to understand the standards better and to make the test more practicable, this paper will focus on the following technological difficulties: to compare the technical requirements of the clearance and the creepage distance of IEC62115: 2011 Electric toys - Safety and those of GB19865-2005 Electric toys - Safety; to discuss the influence of the pollution degrees and isolation material types on the test results; to determine the calculation rules and testing conditions of clearances and creepage distances in various conditions and to illustrate the calculation rules and measurement paths.

\section{Comparison of Technical Requirements of Creepage Distances and Clearances between IEC 62115 and GB19865}

For the current China standard GB 19865-2005 Electric Toys-Safety, its technical requirements mainly are equivalent to IEC 62115:2003(A1:2004), but for the upcoming release version of GB19865, there will be some big change in chapter 18 Creepage Distances and Clearances, where the technical requirements are mainly equivalent to IEC62115:2011 chapter 18. The following will be some comparison between IEC 62115:2011 and GB 19865 on creepage distances and clearances. 


\section{The Technical Requirements on Creepage Distances and Clearances of GB19865-2005}

Clearances and creepage distances of functional insulation shall not be less than $0.5 \mathrm{~mm}$ except when the toy meets the requirements of Clause 9 with this distance short circuited.

\section{The Technical Requirements on Creepage Distances and Clearances of GB 19865-2005}

Besides the technical requirements of GB19865-2005 mentioned above, the technical requirements of IEC62115 also include: However, for functional insulation on printed circuit boards, except at their edges, this distance may be reduced to $0.2 \mathrm{~mm}$ provided that the degree of pollution in the microenvironment in which the insulation is located is unlikely to exceed pollution degree 2 during the normal use of the toy.

Internal parts of toys that comply with subclause 14.15 and have a voltage exceeding $24 \mathrm{~V}$ shall have clearances and creepage distances for functional insulation equal to or greater than the values in Table 18 of IEC 60335-1 for pollution degree 2 except when the toy meets Clause 9 with this distance short circuited.[1]

\section{Technical Requirements Comparison of Creepage Distance and Clearance in IEC62115 and GB19865}

There are some big changes in IEC62115:2011 compared to IEC 62115:2003(A1:2004) on the technical requirements of creepage distances and clearances, so there are some apparent difference on the technical requirements of creepage distances and clearances in IEC 62115 and GB 19865.

The related relaxation or exemption clauses are added in IEC 62115:2011 for printed circuit boards (PCB) or low power electric toys, considering the less danger and their function. For example, for functional insulation on the printed circuit boards this distance may be reduced to $0.2 \mathrm{~mm}$; No requirements for clearances and creepage distances of functional insulation when the toy meets the requirements of clause 9 with this distance short-circuited. Furthermore, new technical contents on pollution degrees and insulation materials are added into IEC 62115:2011 for electric toys having a voltage exceeding $24 \mathrm{~V}$, which shall have clearances or creepage distances for functional insulation equal to or greater than the values of table 18 of IEC 60335-1 for pollution degree 2 as shown in table 1.The new standard takes the greater hazard of high voltage into account. Table 1shows that the higher the working voltage, the greater the relevant limits, where the minimum limit is $20 \mathrm{~mm}$ for functional insulations having a working voltage exceeding $4000 \mathrm{~V}$ that is far greater than $0.5 \mathrm{~mm}$ (GB19865 limit).

Table 1. Minimum creepage distances for functional insulation (Pollution degree 2)

\begin{tabular}{|c|c|c|c|}
\hline \multirow{2}{*}{ Working voltage(V) } & \multicolumn{3}{|c|}{ Creepage distance(mm) } \\
\cline { 2 - 4 } & \multicolumn{3}{|c|}{ Material group } \\
\cline { 2 - 4 } & I & II & III a/III b \\
\hline$\leq 50$ & 0.56 & 0.8 & 1.1 \\
$>50$ and $\leq 125$ & 0.71 & 1.0 & 1.4 \\
$>125$ and $\leq 250$ & 1.0 & 1.4 & 2.0 \\
$>250$ and $\leq 400$ & 1.6 & 2.2 & 3.2 \\
$>400$ and $\leq 500$ & 2.0 & 2.8 & 4.0 \\
$>500$ and $\leq 800$ & 3.2 & 4.5 & 6.3 \\
$>800$ and $\leq 1000$ & 4.0 & 5.6 & 8.0 \\
$>1000$ and $\leq 1250$ & 5.0 & 7.1 & 10.0 \\
$>1250$ and $\leq 1600$ & 6.3 & 9.0 & 12.5 \\
$>1600$ and $\leq 2000$ & 8.0 & 11.0 & 16.0 \\
$>2000$ and $\leq 2500$ & 10.0 & 14.0 & 20.0 \\
$>2500$ and $\leq 3200$ & 12.5 & 18.0 & 25.0 \\
$>3200$ and $\leq 4000$ & 16.0 & 22.0 & 32.0 \\
$>4000$ and $\leq 5000$ & 20.0 & 28.0 & 40.0 \\
\hline
\end{tabular}

Note: It is regulated that the max working voltage of an electric toy is not greater than 5000V inIEC62115:2011. 


\section{Test Cases Comparison According to IEC62115 and GB19865}

First, the technical requirements of creepage distances and clearance in IEC62115 and GB19865 are compared with two test cases. Figure 1 shows a printed circuit board (PCB). It is measured that the clearance between the positive and the negative of its power is $0.35 \mathrm{~mm}$, which is less than the $\operatorname{limit}(0.5 \mathrm{~mm})$ of GB $19865-2005$ but is greater than the limit $(0.2 \mathrm{~mm})$ of IEC62115:2011, so it is found to comply with the requirement of IEC62115:2011 but not to comply with the requirement of GB19865. It shows that the requirement of creepage distances and clearance in IEC62115: 2011 for PCB is relaxed.

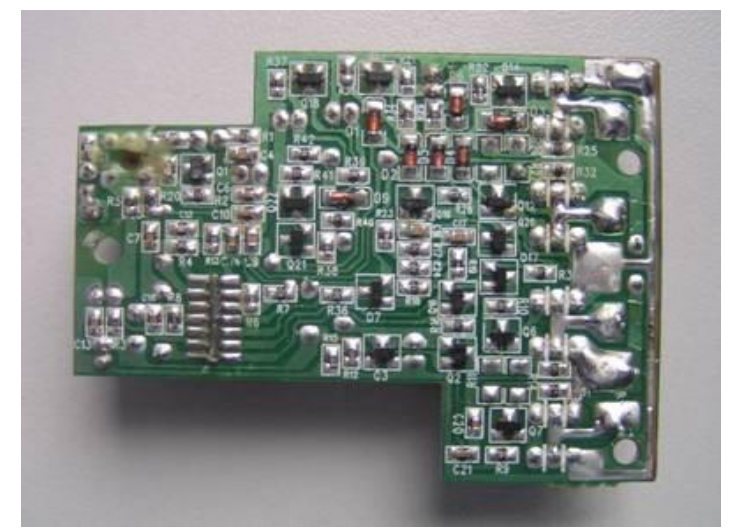

Figure 1. Example of a printed circuit board (PCB)

Figure 2 shows the switch-leg solder pins of an electrical ride-on toy vehicle, where the red leg of the left pin is connected to the positive of the power and the black leg of the middle pin to the negative with an operating voltage $26 \mathrm{~V}$. It is measured that the isolation

clearance between the live parts with different polars is $0.53 \mathrm{~mm}$, which is higher than the limit $(0.5 \mathrm{~mm})$ of GB19865 but lower than the limit $(0.56 \mathrm{~mm})$ of IEC 62115 , so it is found to comply with the requirement of GB 19865 but not to comply with IEC 62115. Therefore the requirement of IEC 62115 for the toy with a voltage exceeding $24 \mathrm{~V}$ is stricter and more practical. The following discussion is mainly on the division of pollution degrees and material groups and the measure path and methods of the creepage distance and clearance correspondingly.

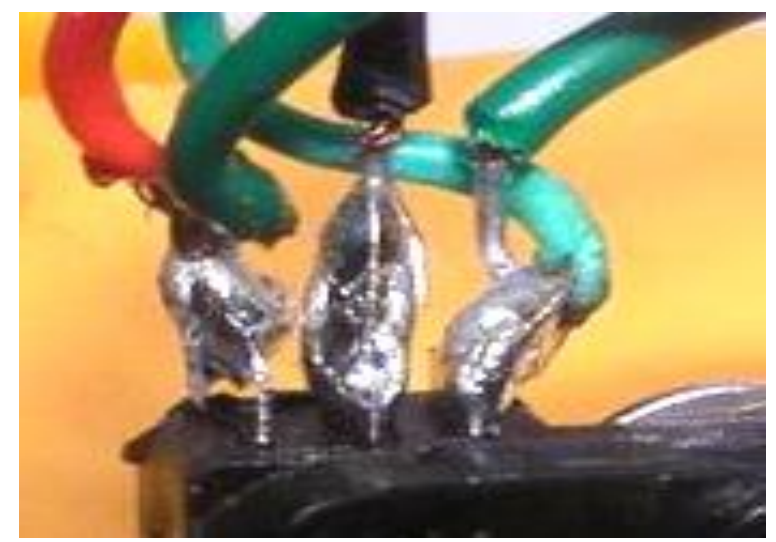

Figure 2. Example of a toy with a voltage exceeding $24 \mathrm{~V}$.

\section{Pollution Degrees}

Pollution is caused by external substances including solid, liquid and gas, to bridge small clearances and reduce the surface resistance of the insulation materials as not to bear the possible maximum transient overvoltage in the circuit. As a result, shells including sealing can reduce 
pollution effectively [2]. According to technical requirements of IEC 62115: 2011, degrees of pollution in the microenvironment are as follows:

- Pollution degree 1: no pollution or only dry, non-conductive pollution occurs. The pollution has no influence;

- Pollution degree 2: only non-conductive pollution occurs, except that occasionally a temporary conductivity caus ed by condensation is to be expected;

- Pollution degree 3: conductive pollution occurs or dry non-conductive pollution occurs that becomes conductive due to condensation that is to be expected;

- Pollution degree 4: the pollution generates persistent conductivity caused by conductive dust or by rain or snow.

Note: Pollution degree 4 is not applicable to appliances.

As for electric toys, pollution degree 1 or 2 are usually determined because the playing environment is good only to cause non-conductive pollution and their electric parts are enclosed totally by shells; but for some electric toys with special function for exam ple electrical toy boat intended to be played in water and washable electric toys, whether the higher pollution degree shall be determined or not is according to the practical conditions. Besides, according to IEC62115:2011, for an electric toy having a voltage exceeding $24 \mathrm{~V}$, its clearance and creepage distances for functional insulation shall be equal to or greater than the value of pollution grade 2 in Table 18 of IEC 60335-1, which is only the requirement for the limit value and shall not have effect on its pollution degree classification.

\section{Material Insulation (Material Group)}

Tracking means the surface of a solid insulating material is destroyed and a conductive path is progressively produced on it due to the combined effects of pollution, leakage current and flash discharge.

The material groups are divided as follows according to the comparative tracking index (CTI) values:

- Material group I: $600 \leq \mathrm{CTI}$

- Material group II: $400 \leq \mathrm{CTI}<600$;

- Material group IIIa: $175 \leq \mathrm{CTI}<400$;

- Material group IIIb: $100 \leq$ CTI $<175$.

The above CTI values are obtained in GB4706.1, where specimens are prepared specially and the specified solution is used in tests to determine CTI values (i.e. numerical value of the maximum voltage at which the material surface can withstand 50 drops of solution without a tracking failure) [3].

For glass, porcelain or other inorganic insulating material will not produce a tracking failure, the creepage distance does not have to be greater than its relevant clearance, which is one reason why this kind of material is chosen as power transmission and transformation equipment material. When designing a creepage distance, it is necessary to arrange some arched-convex and grooves to prevent the leakage current passing to slowing down the progress of tracking failure [2].

According to IEC 62115:2011 clause 18, internal parts of toys that comply with subclause 14.15 and have a voltage exceeding $24 \mathrm{~V}$ shall have clearance and creepage distances for functional insulation equal to or greater than the values in Table 18 of IEC $60335-1$ for pollution degree 2 . So creepage distances of toys that have a voltage exceeding $24 \mathrm{~V}$ but not exceeding $50 \mathrm{~V}$ shall be: material group I: $0.5 \mathrm{~mm}$; material group II: $0.8 \mathrm{~mm}$; material group IIIa/IIIb: $1.1 \mathrm{~mm}$, according to material groups of table $1^{[3]}$.

\section{Minimum Conductive Distance (Groove Dimension) $\mathbf{X}$}

The conductive distance (groove dimension) $\mathrm{X}$ depends on the pollution degree of different electric toys and if the groove dimension is less than $\mathrm{X}$, it is supposed that the creepage distance is equal to the clearance. The grooves dimensions $X$ specified in examples of part 4 are the minimum values depending on the pollution degrees described in IEC 60664-1, as shown in table 2. If the associated clearance is less than $3 \mathrm{~mm}$, the minimum dimension $X$ may be reduced to $1 / 3$ of this clearance. [4] 
Table 2. Groove dimensions (X) depending on pollution degrees

\begin{tabular}{|c|c|}
\hline Pollution degree & Minimum groove dimension X(mm) \\
\hline 1 & 0.25 \\
\hline 2 & 1 \\
\hline 3 & 1.5 \\
\hline
\end{tabular}

\section{Measurement Paths and Rules}

When mearsuring creepage distances and clearances, the pollution degrees and the minimum groove dimensions $\mathrm{X}$ of the relevant functional insulation of the electric toy shall be took into consideration, according to IEC60664-1, to determine the measurement path and the rule. The creepage distance and the clearance are measured when the parts moving against each other are placed in the most unfavorable position. The measurement method is shown in the following examples, where the dotted line ----- is the clearance and the hatching line creepage distance ${ }^{[4]}$. For toys having a voltage exceeding $24 \mathrm{~V}$, creepage distances and clearances of functional insulation shall be equal to or greater than the values in Table 18 of IEC 60335-1 for the minimum creepage distance of functional insulation (pollution degree 2, see table 1); namely creepage distance limits are equal to clearance limits at the same working voltage. With the following examples, it is proved that the creepage distance must be greater than or equal to the clearance, so in the practical measurement, it is only the clearance that shall be measured.

\section{Example of Paths with Concave Grooves}

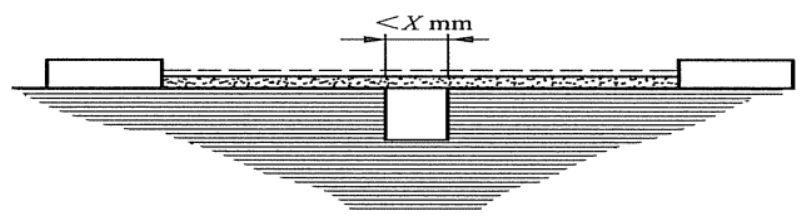

Figure 3-1 Example that the concave groove may be ignored

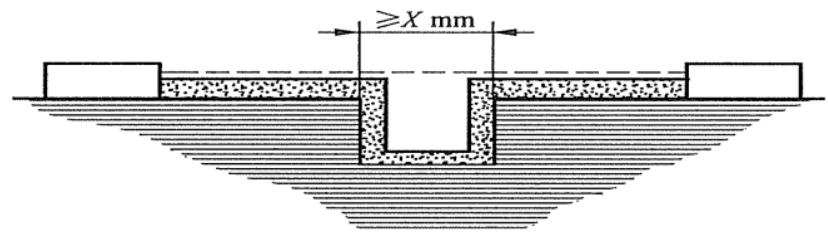

Figure 3-2 Example that the concave groove shall be considered

The path under consideration as shown in Figure 3-1 includes a parallel- or converging-sided groove of any depth with a width less than $\mathrm{X} \mathrm{mm}$, because the width is less than corresponding requirement of the pollution degree, and the creepage distance and clearance are measured directly across the groove as shown, where the creepage distance is equal to the clearance. The path under consideration as shown in Figure 3-2 includes a parallel-sided groove of any depth and equal to or more than $\mathrm{Xmm}$, because the width is more than the corresponding requirement of the pollution degree, so the clearance is the "line of sight" distance but the creepage path follows the contour of the groove, where the creepage distance is greater than the clearance.

\section{Example of a Path with a V-Shaped Groove}

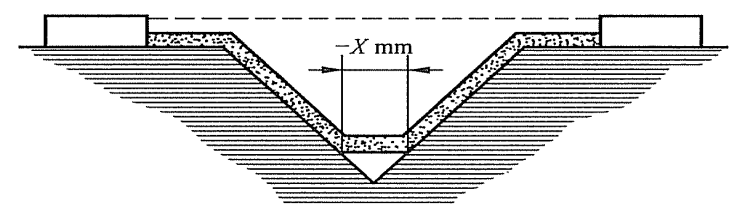

Figure 4 Example of a V-shaped groove 
The path under consideration as shown in photo 4 includes a V-shaped groove with a width greater than X mm. The clearance is the "line of sight" distance and the creepage path follows the contour of the groove but "short-circuits" the bottom of the groove by $\mathrm{X}$ mm link.

\section{Examples of Paths with Heads of Screws}

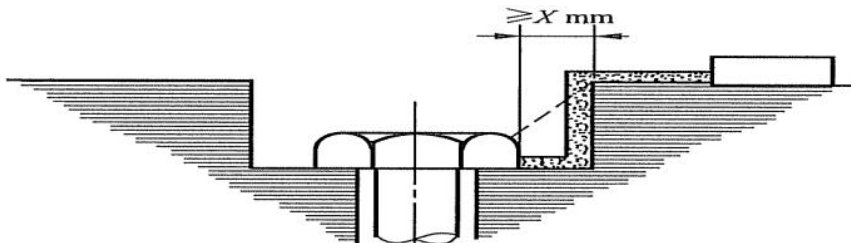

Figure 5-1, Example that the gap between the head of the the screw and the wall is $\geq \mathrm{Xmm}$

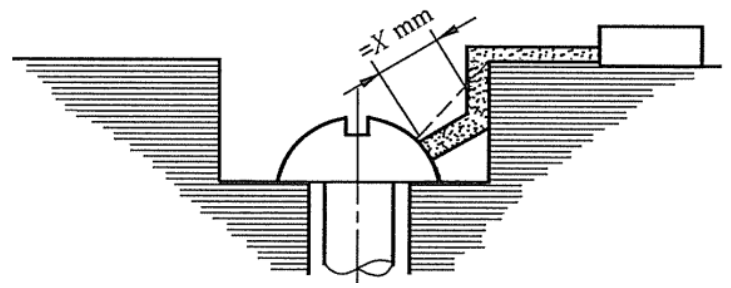

Figure 5-2, Example that the gap between the head of the screw and the wall is $<\mathrm{Xmm}$

When the gap between the head of the screw and the wall is $\geq X \mathrm{Xm}$ as shown in photo $5-1$, the creepage distance is the contour of the groove and the clearance is shown as "----"

When the gap between the head of the screw and the wall is $<\mathrm{Xmm}$ as shown in photo 5-2, the creepage distance is from the screw to the wall when the distance is equal to $\mathrm{X} \mathrm{mm}$ and the clearance is shown as "----"

\section{Conclusion}

It is shown that 1. Exemption conditions for a printed circuit board (PCB) or a low power electric toy are more relaxed in IEC 62115:2011 considering its less hazard and its function; 2. The technical requirements are stricter for toys having a voltage exceeding $24 \mathrm{~V}$ considering its more hazard with comparing and analyzing the technical requirements of creepage distances and clearances in IEC 62115:2011 and GB19865-2005. Starting with the theory of creepage distances and clearances, factors having effects on creepage distances and clearances including pollution degrees and material insulation are analyzed and measurement paths and rules are studied. All above are expected to be helpful for testing engineers in understanding and improving their tests.

\section{References}

1. GB 19865-2005 Electric toys - Safety, IEC62115: 2011Electric toys - Safety

2. Deng Zhenjin Creepage distances and clearances in GB4793.1 -2007. Medical Equipment, 2011, 01:9-12

3. IEC 60335-1: 2010 Household and similar electrical appliances - Safety - Part 1: General Requirements

4. IEC 60664-1:2007 Insulation coordination for equipment with low-voltage system Part 1:Principles requirements and tests 